\title{
Chemical profiling, antimicrobial and insecticidal evaluations of Polygonum hydropiper $\mathrm{L}$
}

Muhammad Ayaz ${ }^{1 *}$, Muhammad Junaid ${ }^{1}$, Farhat Ullah¹, Abdul Sadiq ${ }^{1}$, Muhammad Ovais², Waqar Ahmad ${ }^{1}$, Sajjad ahmad ${ }^{1}$ and Anwar Zeb ${ }^{1}$

\begin{abstract}
Background: The emergence of multidrug resistant (MDR) pathogens is of great concern to the global health community. Our ability to effectively treat diseases is based on the discovery of potent drugs for the treatment of these challenging diseases. Traditional medicines are one of the major sources for the discovery of safe, effective and economical drug candidates. In order to validate its antibacterial, antifungal and insecticidal potentials with respect to traditional uses, we have screened for the first time Polygonum hydropiper against pathogenic bacterial, fungal strains and a variety of insects.
\end{abstract}

Methods: Polygonum hydropiper samples including crude extract (Ph.Cr), subsequent fractions; $n$-hexane (Ph.Hex), chloroform (Ph.Chf), ethyl acetate (Ph.EtAc), n-Butanol (Ph.Bt), aqueous (Ph.Aq) and crude saponins (Ph.Sp) were tested against pathogenic bacterial and fungal strains. Insecticidal activities were performed against Tribolium castaneum and Rhyzopertha dominica and Monomorium pharaonis. Ph.Cr was analyzed by gas chromatography-mass spectrometry (GC-MS) for preliminary identification of chemical constituents.

Results: In disc diffusion assay, Ph.Chf, Ph.Hex, Ph.EtAc and Ph.Sp exhibited highest activity against Enterococcus faecalis. MICs of Ph.Chf against Enterococcus faecalis, Klebsiella pneumoniae, Escherichia coli, P. mirabilis, Staphylococcus aureus, Salmonella typhi and Pseudomonas aeruginosa were 32.00, 13.33, 10.66, 5.33, 64.00, 8.66 and $10.66 \mu \mathrm{g} / \mathrm{ml}$ respectively. MFC's of Ph.Chf against Aspergillus fumigatus, Aspergillus flavus, Aspergillus niger and Fusarium oxysporum were 16.66, 23.33, 125.00 and $46.66 \mu \mathrm{g} / \mathrm{ml}$ respectively. Ph.EtAc, Ph.Sp, Ph.Chf and Ph.Bt were most active fractions against $T$. castaneum and $R$. dominica. Ph.Sp being most active against $A$. punctatum exhibited $L C_{50}$ of $<0.01 \mathrm{mg} / \mathrm{ml}$. In GC-MS analysis of Ph.Cr, 124 compounds were identified among which several bioactive antibacterial, antifungal and insecticidal compounds were found.

Conclusions: $P$. hydropiper samples exhibited broad spectrum of activity against bacterial and fungal strains. Our results support previously reported insecticidal properties of saponins and may provide scientific justification for the ethno-medicinal uses of the plant.

Keywords: Tribolium castaneum, Rhyzopertha dominica, Monomorium pharaonis, Anobium punctatum, Larvicidal, Gas chromatography, Fungicidal and antibacterial activity

\footnotetext{
* Correspondence: ayazuop@gmail.com

${ }^{1}$ Department of Pharmacy, University of Malakand, Khyber Pakhtoonkhwa

(KPK) 18000, Pakistan

Full list of author information is available at the end of the article
} 


\section{Background}

The emergence of multidrug resistant (MDR) pathogens and life-threatening infections caused by these microorganisms is a global challenge for scientific community and some scientists speculate that we are going back to the pre-antibiotic era $[1,2]$. The prevalence of microbial infections due to opportunistic pathogens, frequently characterized by high mortality rates, has increased during the past two decades [3]. Majority of antibiotics, currently applied in therapy, belong to drug classes discovered prior to 1970 [4]. The current expansion of antibacterial and antifungal drugs research has occurred since there is persistent need for developing new compounds to fight life-threatening infections [5]. Besides bacterial infections, fungi are the major causes of liver, lungs, mouth, blood and skin infections [6]. Systemic mycoses are most frequently caused by Candida genus yeasts and mould particularly, the Aspergillus genus. Moreover, many of the existing drugs are toxic, ineffective and enable infection recurrence because of being bacteriostatic/fungistatic in nature. Medicinal plants are potential sources of potent antimicrobial drugs and are used in many countries to treat infectious diseases [7]. Over the years, traditional phytotherapy is in practice for the treatment of microbial and non-microbial origin diseases [8]. World Health Organization (WHO) estimates that approximately $80 \%$ population of underdeveloped countries rely on medicinal plants for their primary health care [9].

Globally, researchers are trying to increase food production to fulfill the excessive food demand due to growing population. Unfortunately, insects are major contributors to extensive qualitative and quantitative loss of food grains, their products, and economically important crops. A total of $10-40 \%$ loss of food grains has been estimated due to insects globally. In spite of improved storage structures and traditional control practices, $70-90 \%$ of food grain cannot be stored for more than 6-12 months at farmer's level [10]. Consequently, there is an immense need to employ safe insecticidal drugs and repellents to protect food grains from damages. In this regard, synthetic insecticidal agents are useful but their uses are limited due to development of insects' resistance, high cost and deposition of toxic residue on grains. Therefore, there is a dire need to develop economical, safe, environment friendly and more effective insecticidal agents.

Approximately, fifteen hundred insecticidal plants has been reported among which ryania, nicotine, rotenone, sabadilla, pyrethrin and azadirachtin are commercially available [11]. T. castaneum (flour beetle) and Rhyzopertha dominica (grain borer) are common insect pests for food processing facilities such as mills, processing plants, warehouses and retail stores [12]. Both these insects have a long association with human stored foods and are commonly found in grain, cereal products, flour, peas, beans, nuts, dried fruits and spices [13]. Anobium punctatum, commonly known as woodworm or furniture beetle, is a common cause of damage to timber worldwide. During the last five decades, insecticidal treatments are extensively employed to minimize the insects' risk especially for the timbers in buildings [14]. Besides this, Monomorium pharaonis is the main cause of damage to food stuff, store grains and wood products.

$P$. hydropiper is traditionally used to treat inflammation, gastrointestinal disturbances, neurological disorders and diarrhea [15]. Plant decoctions are used to treat an extensive range of ailments like dyspepsia, diarrhea, menorrhagia, hemorrhoids and skin itching [16]. Recently, $P$. hydropiper has been reported for anticholinesterase, antioxidant, phytotoxic, anthelmintic and anti-cancer potentials [17-20]. The current study was aimed to uncover the antibacterial, antifungal and insecticidal potentials of $P$. hydropiper extracts and sponins.

\section{Methods \\ Plant collection, extraction and fractionation}

P. hydropiper aerial parts (stem, leaves and flowers) were collected from Talash Valley, Khyber Pakhtoonkhwa, Pakistan in July, 2013. The plant was identified by Dr. Gul Rahim, botanical taxonomist. A sample was deposited at the herbarium, University of Malakand Chakdara (Dir), Pakistan with voucher no (H.UOM.BG.107). Plant material was cleansed, shade dried for 15 days and coarsely crushed with a cutter mill. Crude powder (4.5 kg) was soaked in $22 \mathrm{~L}$ of $80 \%$ methanol for 10 days with frequent shaking. This extraction with methanol was three times followed by filtration from muslin cloth [21]. The filtrate was concentrated using rotary evaporator (Heidolph Laborota 4000, Schwabach, Germany) under reduced pressure at $40{ }^{\circ} \mathrm{C}$, which resulted in $290 \mathrm{~g}(6.44 \%)$ of dark brown colored crude extract [22]. Ph.Cr (250 g) was processed for fractionation purpose following procedure we reported previously [17].

\section{Extraction of crude saponins}

Saponins were extracted from $60 \mathrm{~g}$ of powdered plant material following our previously reported procedure [17]. Finally, $9 \mathrm{~g}$ of saponins with a percent yield of $15 \%$ were obtained.

Gas chromatography-mass spectrometry (GC/MS) analysis Ph.Cr was analyzed by means of an Agilent USB-393752 gas chromatograph (Agilent Technologies, Palo Alto, CA, USA) with HHP-5MS 5\% phenylmethylsiloxane capillary column $(30 \mathrm{~m} \times 0.25 \mathrm{~mm} \times 0.25 \mu \mathrm{m}$ film thickness Restek, Bellefonte, PA) equipped with an flame ionization (FID) detector. Helium was used as carrier gas at a flow rate of $1 \mathrm{ml} / \mathrm{min}$, and diluted samples (1/1000 in $n$-pentane, $\mathrm{v} / \mathrm{v})$ 
of $1.0 \mu \mathrm{l}$ were injected manually in the splitless mode. GC/ MS analysis of Ph.Cr was processed using an Agilent USB-393752 gas chromatograph (Agilent Technologies, Palo Alto, CA, USA) with a HHP-5MS 5\% phenylmethylsiloxane capillary column $(30 \mathrm{~m} \times 0.25 \mathrm{~mm} \times 0.25 \mu \mathrm{m}$ film thickness Restek, Bellefonte, PA) outfitted with an Agilent HP-5973 mass selective detector in the electron impact mode (Ionization energy: $70 \mathrm{eV}$ ) working under the same experimental conditions as described for GC [23].

\section{Chemical and drugs}

Nutrient agar (Oxoid Ltd, UK), Nutrient broth (Oxoid), Sabouraud's dextrose agar (SDA), Dimethyl-Sulfoxide DMSO (Labscan Patumwan Bankok 10330 Thialand), Permethrin (CAS 52645-53-1) Sigma aldrich laborchemikalie $\mathrm{GmbH}$, ceftriaxone (Geltis, Shaigan Pharmaceuticals), antibiotic discs (Oxoid) of ciprofloxacin, moxifloxacin, amoxicillin and gentamicin, amphotericin-B were used in the study. Solvents used were of analytical grade and were purchased from authorized dealer of Sigma Aldrich CHEMIE GmbH USA, Pakistan.

\section{Collection and identification of bacteria}

Bacterial strains including Staphylococcus aureus (29213), Enterococcus faecalis (29212), Klebsiella pneumoniae (700603), Escherichia coli (739), Proteus mirabilis (13315), Salmonella typhi and Pseudomonas aeruginosa (27853) were used in the study. Bacterial strains were provided by Department of Microbiology, Quaid-i-Azam University Islamabad Pakistan. These strains were identified by different biochemical tests and were preserved in freeze-dried condition at $4{ }^{\circ} \mathrm{C}$ in stab slant agar until later use [24].

\section{Standardization of bacterial suspension}

Bacterial cultures were grown for $24 \mathrm{~h}$ at $37{ }^{\circ} \mathrm{C}$ and suspension with cell density of $1 \times 10^{8} \mathrm{CFU} / \mathrm{ml}$, were prepared using McFarland standard and were further diluted to a cell density of $1 \times 10^{6} \mathrm{CFU} / \mathrm{ml}$ using a UV visible spectrophotometer (Thermo electron corporation USA) at $625 \mathrm{~nm}$. The standardization was maintained for the whole period of the study.

\section{Antibacterial investigations \\ Bacterial susceptibility pattern}

Susceptibility pattern of selected bacterial strains was determined by disc diffusion method using standard antibiotic discs of ceftriaxone, ciprofloxacin, moxifloxacin, amoxicillin and gentamicin. Diameter of inhibitory zones indicated sensitivity or resistance to these antibiotics.

\section{Disc diffusion assay}

For determination of antibacterial potential of plant extracts, a qualitative to semi quantitative disc method was used following previously reported procedure [5]. Briefly, nutrient agar plates, prepared aseptically, were inoculated with test organisms under laminar flow hood. Sterile paper discs of $6 \mathrm{~mm}$ diameter (Whatman International, CAT: 2017-006) impregnated with different concentrations (25, $50,100 \mu \mathrm{g} / \mathrm{ml}$ ) of extracts were placed equidistantly onto the surface of the already inoculated Petri dishes using sterile forceps. Blank discs impregnated with DMSO/solvents were used as negative control whereas, ceftriaxone discs $(25,50,100 \mu \mathrm{g} / \mathrm{ml})$ were used as positive control. The plates were incubated at $37{ }^{\circ} \mathrm{C}$ for $24 \mathrm{~h}$ and zone of inhibition was measured around the discs.

Determination of Minimum Inhibitory Concentrations (MICs) For determination of MICs, both broth and agar dilution methods approved by clinical and laboratory standard institute (CLSI) were used $[25,26]$. For these tests, plant extracts in serial dilutions of $2-512 \mu \mathrm{g} / \mathrm{ml}$ were added to sterilized tube containing nutrient broth, so that the final concentration of the test samples were $2-512 \mu \mathrm{g} / \mathrm{ml}$. Tubes were inoculated with the test microbes. Tubes were incubated using shaker incubator at $37{ }^{\circ} \mathrm{C}$ for $24 \mathrm{~h}$.

\section{Antifungal investigations}

Fungal strains Four fungal strains including A. fumigatus, $A$. niger, $A$. flavus and $F$. oxysporum were used to determine antifungal potential of plant extracts. Fungal strains were kindly provided by Department of Microbiology, Quaid-i- Azam University Islamabad Pakistan.

Preliminary antifungal activity Before proceeding to detail antifungal studies, antifungal potential of all samples were performed. Briefly, each plant sample was prepared at concentration of $10 \mathrm{mg} / \mathrm{ml}$ and one $\mathrm{ml}$ was added to $9 \mathrm{ml} \mathrm{SDA}$, already prepared in test tubes. These test tubes were inoculated with the fungal strains and were incubated at $27{ }^{\circ} \mathrm{C}$ for 7 days. Finally, test tubes were checked for inhibition of fungal growth [27].

Disc diffusion assay Antifungal potentials of $P$. hydropiper extracts and spaonins were investigated by disc diffusion method as previously reported [28, 29]. Sabouraud dextrose Agar (SDA) plates were prepared and inoculated with the test fungi under laminar flow hood. Sterile paper discs of $6 \mathrm{~mm}$ diameter (Whatman International, CAT: 2017-006), impregnated with different concentrations of extracts and standard drug (125, 250 and $500 \mu \mathrm{g} / \mathrm{ml})$ were placed equidistantly onto the surface of these Petri dishes and were incubated at $27{ }^{\circ} \mathrm{C}$ for $72 \mathrm{~h}$. Diameter of Inhibitory zone around the discs was measured and was compared with standard drug.

Minimum Fungicidal Concentration (MFCs) Minimum fungicidal concentrations (MFCs) of plant extracts were 
determined using agar dilution techniques in Sabouraud's dextrose agar (SDA) and nutrient broth. SDA and nutrient broth (Oxoid Ltd, England) were prepared according to manufacturer specifications and serial dilutions of samples $2.5-1000 \mu \mathrm{g} / \mathrm{ml}$ were aseptically added to these tubes at $40{ }^{\circ} \mathrm{C}$. The tubes were inoculated by adding one loopful of already prepared fungal suspensions and were incubated at $27^{\circ} \mathrm{C}$. After 7-10 days, tubes were observed for fungal growth and MFCs were considered the lowest concentration which inhibited fungal growth [27].

Collection and identification of the insects The Tribolium castaneum (flour beetle) was collected from the flour mill Chakdara in the proximity of University of Malakand. Similarly, the Rhyzopertha dominica (grain borer) was collected from the grocery shop in university town, University of Malakand. Anobium punctatum (wood worm) was collected from timber market Chakdara. Likewise, the Monomorium pharaonis (Pharaoh ants) were collected from the main campus, University of Malakand. All the insects were identified and authenticated by Saeed Ahmad, Assistant Professor, Department of Zoology, University of Malakand.

Insecticidal activity against $T$. castaneum and $R$. dominica Insecticidal potential of plant extracts was tested on adult insects of $T$. castaneum and $R$. dominica, using previously reported procedure [30]. Different concentrations $(125-500 \mu \mathrm{g} / \mathrm{ml})$ of plant extracts were prepared in methanol. Filter papers were dipped in these solutions and were transferred to sterile Petri dishes. The plates were left overnight for evaporation of the solvent. Thirty healthy and active insects of both species were transferred to test group Petri dishes, positive control (Permethrin) and negative control groups Petri dishes and were kept in growth chamber at $27^{\circ} \mathrm{C}$ for $24 \mathrm{~h}$ with $50 \%$ relative humidity. Percent insecticidal activity was determined from the number of dead insects after 24 and $48 \mathrm{~h}$.

Anti-anobium investigations The anti-anobium activity of Plant samples was evaluated following previously reported procedure [31]. Using this procedure, different plant extracts were assayed for lethality against $A$. punctatum. Briefly, different concentrations of samples were prepared by dissolving $100 \mathrm{mg} / \mathrm{ml}$ in respective solvents and were further diluted. Sterilized filter papers were put in sterile Petri dishes. Solvents were transferred to Petri dishes and kept overnight for the removal of solvents. A. punctatum, 25 larva's were transferred to each Petri dish and were kept at room temperature for $24 \mathrm{~h}$. The number of dead and alive larva were counted

Anti-pharaoh investigations Anti-Pharaoh potential of samples were determined by contact toxicity method, following previously reported procedure [32]. Sample solutions in concentrations of $12.5-50 \mathrm{mg} / \mathrm{ml}$ were added to sterile Petri dishes containing filter paper and were left overnight for evaporation of solvents. Thereafter, 30 pharaohs were transferred to each Petri dish and were incubated at room temperature for $24 \mathrm{~h}$. Finally, the numbers of dead and alive Pharaoh were counted in each Petri dish. The Petri dishes containing filter paper plus distilled water served as a control.

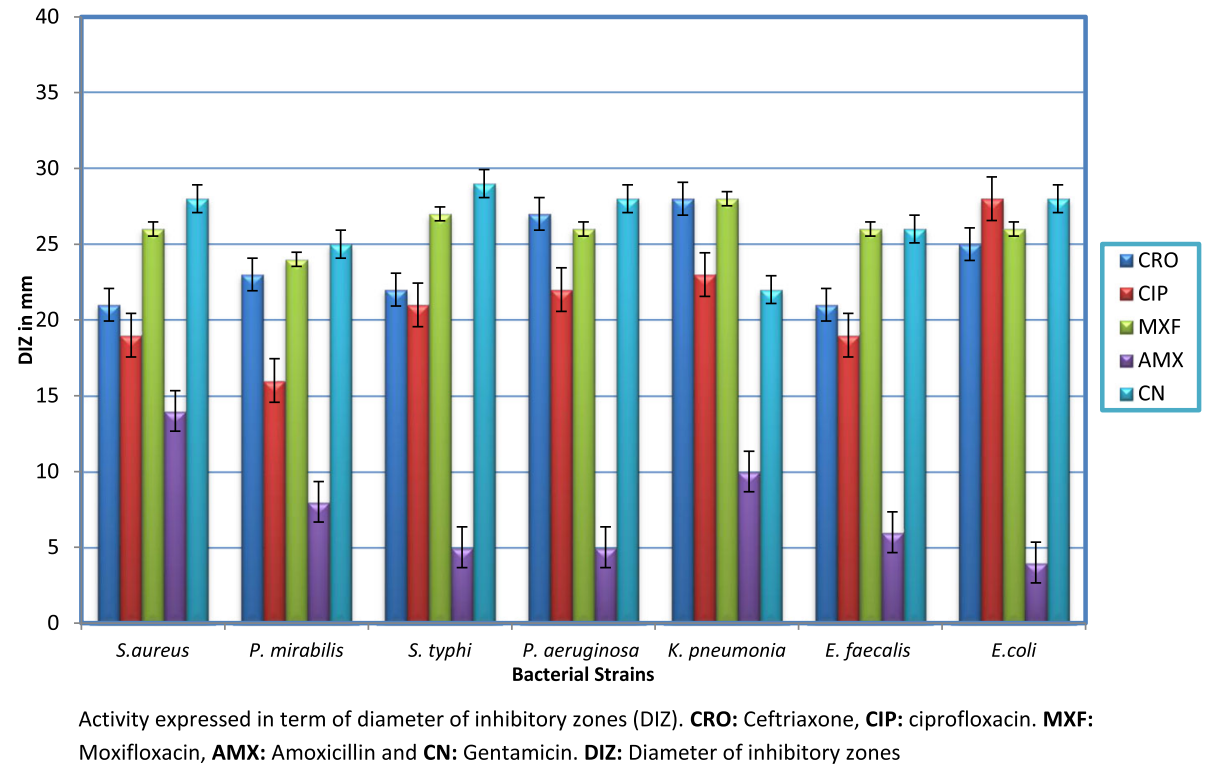

Fig. 1 Susceptibility pattern of bacterial strains exposed to standard antibiotic discs 
Table 1 Antibacterial activity of Polygonum hydropiper extracts and saponins against bacterial strains

\begin{tabular}{|c|c|c|c|c|c|c|c|c|}
\hline \multirow[t]{2}{*}{ Samples } & \multirow{2}{*}{$\begin{array}{l}\text { Conc. } \\
\mu \mathrm{g} / \mathrm{ml}\end{array}$} & \multicolumn{7}{|c|}{ Diameter of the inhibitory zone $(\mathrm{mm})$ Mean $\pm \operatorname{SEM}(n=3)$} \\
\hline & & $\begin{array}{l}\text { Enterococcus } \\
\text { faecalis }\end{array}$ & $\begin{array}{l}\text { Klebsiella } \\
\text { pneumonia }\end{array}$ & Escherichia coli & $\begin{array}{l}\text { Proteus } \\
\text { mirabilis }\end{array}$ & $\begin{array}{l}\text { Staphylococcus } \\
\text { aureus }\end{array}$ & $\begin{array}{l}\text { Salmonella } \\
\text { typhi }\end{array}$ & $\begin{array}{l}\text { Pseudomonas } \\
\text { aeruginosa }\end{array}$ \\
\hline \multirow[t]{3}{*}{ Crude (Ph.Cr) } & 25 & $5.33 \pm 0.88$ & $6.33 \pm 0.66$ & $0.00 \pm 0.00$ & $11.00 \pm 0.00$ & $10.00 \pm 0.57$ & $11.33 \pm 1.20$ & $15.66 \pm 1.85$ \\
\hline & 50 & $14.00 \pm 0.57$ & $10.33 \pm 0.33$ & $0.00 \pm 0.00$ & $17.33 \pm 0.88$ & $14.33 \pm 0.33$ & $14.00 \pm 0.57$ & $20.33 \pm 0.88$ \\
\hline & 100 & $17.33 \pm 0.88$ & $14.00 \pm 0.00$ & $9.00 \pm 0.57$ & $34.00 \pm 1.15$ & $18.66 \pm 0.66$ & $21.33 \pm 0.88$ & $25.33 \pm 1.45$ \\
\hline \multirow[t]{3}{*}{ Ethyl acetate (Ph.EtAc } & 25 & $9.33 \pm 0.33$ & $0.00 \pm 0.00$ & $0.00 \pm 0.00$ & $11.66 \pm 1.20$ & $8.33 \pm 0.88$ & $8.00 \pm 0.57$ & $13.00 \pm 0.57$ \\
\hline & 50 & $18.33 \pm 0.88$ & $7.00 \pm 0.57$ & $8.33 \pm 0.88$ & $18.33 \pm 0.66$ & $13.66 \pm 1.20$ & $15.66 \pm 0.66$ & $18.33 \pm 0.66$ \\
\hline & 100 & $27.00 \pm 0.57$ & $13.33 \pm 0.88$ & $15.00 \pm 0.00$ & $27.66 \pm 2.18$ & $18.33 \pm 0.88$ & $20.73 \pm 1.00$ & $24.00 \pm 0.57$ \\
\hline \multirow[t]{3}{*}{ Chloroform (Ph.Chf) } & 25 & $13.66 \pm 0.33$ & $0.00 \pm 0.00$ & $8.00 \pm 0.57$ & $12.66 \pm 3.40$ & $7.33 \pm 0.88$ & $17.33 \pm 0.66$ & $18.66 \pm 1.20$ \\
\hline & 50 & $19.00 \pm 0.57$ & $8.00 \pm 0.57$ & $14.33 \pm 0.33$ & $15.33 \pm 2.02$ & $11.66 \pm 1.45$ & $23.00 \pm 0.57$ & $23.00 \pm 1.00$ \\
\hline & 100 & $31.66 \pm 1.20$ & $15.60 \pm 1.15$ & $18.00 \pm 1.52$ & $21.00 \pm 1.73$ & $16.00 \pm 1.15$ & $29.33 \pm 0.88$ & $31.66 \pm 2.18$ \\
\hline \multirow[t]{3}{*}{ Butanol (Ph.Bt) } & 25 & $7.00 \pm 1.15$ & $0.00 \pm 0.00$ & $0.00 \pm 0.00$ & $14.00 \pm 0.57$ & $13.66 \pm 1.20$ & $11.33 \pm 0.66$ & $7.33 \pm 0.88$ \\
\hline & 50 & $11.00 \pm 1.15$ & $0.00 \pm 0.00$ & $0.00 \pm 0.00$ & $19.66 \pm 1.20$ & $18.66 \pm 1.45$ & $14.00 \pm 1.52$ & $12.66 \pm 0.66$ \\
\hline & 100 & $21.00 \pm 0.00$ & $8.00 \pm 0.00$ & $11.33 \pm 0.88$ & $29.00 \pm 0.00$ & $23.00 \pm 1.15$ & $19.00 \pm 0.57$ & $16.00 \pm 1.52$ \\
\hline \multirow[t]{3}{*}{ n-Hexane (Ph.Hex) } & 25 & $11.66 \pm 0.66$ & $0.00 \pm 0.00$ & $0.00 \pm 0.00$ & $0.00 \pm 0.00$ & $11.00 \pm 1.15$ & $8.66 \pm 1.66$ & $12.66 \pm 0.33$ \\
\hline & 50 & $17.66 \pm 1.66$ & $9.66 \pm 0.33$ & $7.66 \pm 1.20$ & $9.33 \pm 0.66$ & $17.33 \pm 2.18$ & $14.00 \pm 1.00$ & $17.00 \pm 0.57$ \\
\hline & 100 & $30.00 \pm 0.57$ & $15.00 \pm 0.57$ & $12.00 \pm 0.57$ & $14.00 \pm 0.57$ & $26.33 \pm 0.88$ & $20.33 \pm 0.88$ & $21.00 \pm 0.00$ \\
\hline \multirow[t]{3}{*}{ Saponins (Ph.Sp) } & 25 & $6.60 \pm 1.15$ & $0.00 \pm 0.00$ & $11.00 \pm 1.00$ & $0.00 \pm 0.00$ & $10.66 \pm 2.02$ & $22.00 \pm 0.57$ & $15.00 \pm 0.00$ \\
\hline & 50 & $10.33 \pm 1.45$ & $00.00 \pm 0.00$ & $16.00 \pm 0.57$ & $0.00 \pm 0.00$ & $15.00 \pm 0.57$ & $26.33 \pm 0.33$ & 22. $33 \pm 1.20$ \\
\hline & 100 & $26.66 \pm 1.20$ & $7.33 \pm 0.88$ & $23.00 \pm 0.00$ & $8.66 \pm 1.20$ & $22.00 \pm 1.52$ & $30.66 \pm 1.20$ & $27.00 \pm 0.57$ \\
\hline \multirow[t]{3}{*}{ Aqueous (Ph.Aq) } & 25 & $4.00 \pm 0.00$ & $0.00 \pm 0.00$ & $6.00 \pm 1.15$ & $0.00 \pm 0.00$ & $0.00 \pm 0.00$ & $0.00 \pm 0.00$ & $6.33 \pm 0.66$ \\
\hline & 50 & $13.00 \pm 0.57$ & $0.00 \pm 0.00$ & $10.33 \pm 0.33$ & $0.00 \pm 0.00$ & $7.00 \pm 0.57$ & $10.33 \pm 0.66$ & $10.88 \pm 0.33$ \\
\hline & 100 & $20.33 \pm 0.33$ & $10.33 \pm 1.45$ & $26.66 \pm 1.20$ & $11.33 \pm 1.45$ & $14.33 \pm 2.02$ & $15.60 \pm 1.15$ & $13.00 \pm 0.66$ \\
\hline \multirow[t]{3}{*}{ Positive Control } & 25 & $19.33 \pm 2.02$ & $24.00 \pm 0.57$ & $23.33 \pm 1.45$ & $22.57 \pm 0.57$ & $16.66 \vee 0.88$ & $20.33 \pm 0.88$ & $22.33 \pm 0.66$ \\
\hline & 50 & $25.00 \pm 0.57$ & $29.33 \pm 0.66$ & $29.66 \pm 1.20$ & $27.66 \pm 0.88$ & $23.33 \pm 1.52$ & $27.00 \pm 0.57$ & $29.00 \pm 0.57$ \\
\hline & 100 & $31.66 \pm 1.45$ & $35.33 \pm 0.88$ & $34.66 \pm 0.88$ & $31.00 \pm 1.52$ & $27.00 \pm 1.15$ & $34.66 \pm 1.20$ & $36.66 \pm 1.20$ \\
\hline
\end{tabular}

Results expressed as diameter of inhibitory zones (DIZ). Each value represent Mean \pm SEM of three independent experimental results. Positive Control: Ceftriaxone

Table 2 Minimum Inhibitory concentrations (MICs) of solvent extracts from Polygonum hydropiper against bacterial strains

\begin{tabular}{|c|c|c|c|c|c|c|c|c|}
\hline \multirow[t]{2}{*}{ Bacterial strains } & \multicolumn{8}{|c|}{ Minimum inhibitory concentrations (MICs) in $\mu \mathrm{g} / \mathrm{ml}$. } \\
\hline & Crude (Ph.Cr) & $\begin{array}{l}\text { n-Hexane } \\
\text { (Ph.Hex) }\end{array}$ & $\begin{array}{l}\text { Ethyl acetate } \\
\text { (Ph.EtAc) }\end{array}$ & $\begin{array}{l}\text { Butanol } \\
\text { (Ph.Bt) }\end{array}$ & $\begin{array}{l}\text { Chloroform } \\
\text { (Ph.Chf) }\end{array}$ & $\begin{array}{l}\text { Aqueous } \\
\text { (Ph.Aq) }\end{array}$ & $\begin{array}{l}\text { Saponins } \\
\text { (Ph.Sp) }\end{array}$ & Ceftriaxone \\
\hline Enterococcus faecalis & $128.00 \pm 0.00$ & $64.00 \pm 0.00$ & $26.66 \pm 5.33$ & $21.33 \pm 5.33$ & $32.00 \pm 0.00$ & $>512$ & $10.66 \pm 2.66$ & $8.00 \pm 0.00$ \\
\hline $\begin{array}{l}\text { Klebsiella } \\
\text { pneumoniae }\end{array}$ & $53.33 \pm 10.66$ & $42.66 \pm 10.66$ & $13.33 \pm 2.66$ & $26.66 \pm 5.33$ & $13.33 \pm 2.66$ & $256.00 \pm 0.00$ & $32.00 \pm 0.00$ & $4.00 \pm 0.00$ \\
\hline Escherichia coli & $64.00 \pm 0.00$ & $128.00 \pm 0.00$ & $64.00 \pm 0.00$ & $\begin{array}{l}128.00 \pm \\
0.00\end{array}$ & $10.66 \pm 2.66$ & $>512$ & $26.66 \pm 5.33$ & $4.00 \pm 0.00$ \\
\hline Proteus mirabilis & $21.33 \pm 5.33$ & $256.00 \pm 0.00$ & $32.00 \pm 0.00$ & $\begin{array}{l}128.00 \pm \\
0.00\end{array}$ & $5.33 \pm 1.33$ & $128.00 \pm 0.00$ & $6.66 \pm 1.33$ & $8.00 \pm 0.00$ \\
\hline $\begin{array}{l}\text { Staphylococcus } \\
\text { aureus }\end{array}$ & $512.00 \pm 0.00$ & $128.00 \pm 0.00$ & $512.00 \pm 0.00$ & $>512$ & $64.00 \pm 0.00$ & $256.00 \pm 0.00$ & $128.00 \pm 0.00$ & $16.00 \pm 0.00$ \\
\hline Salmonella typhi & $64.00 \pm 0.00$ & $128.00 \pm 0.00$ & $64.00 \pm 0.00$ & $13.33 \pm 2.66$ & $8.66 \pm 0.66$ & $128.00 \pm 0.00$ & $53.33 \pm 10.66$ & $16.00 \pm 0.00$ \\
\hline $\begin{array}{l}\text { Pseudomonas } \\
\text { aeruginosa }\end{array}$ & $26.66 \pm 5.33$ & $32.00 \pm 0.00$ & $64.00 \pm 0.00$ & $32.00 \pm 0.00$ & $10.66 \pm 2.66$ & $512.00 \pm 0.00$ & $6.66 \pm 1.33$ & $4.00 \pm 0.00$ \\
\hline
\end{tabular}


Table 3 Preliminary antifungal activity of $P$. hydropiper extracts and crude saponins

\begin{tabular}{|c|c|c|c|c|c|c|c|c|}
\hline \multirow[t]{2}{*}{ Fungal strains } & \multicolumn{8}{|c|}{ Inhibition of fungal growth } \\
\hline & $\begin{array}{l}\text { Crude } \\
\text { (Ph.Cr) }\end{array}$ & $\begin{array}{l}\text { n-Hexane } \\
\text { (Ph.Hex) }\end{array}$ & $\begin{array}{l}\text { Ethyl acetate } \\
\text { (Ph.EtAc) }\end{array}$ & $\begin{array}{l}\text { Butanol } \\
\text { (Ph.Bt) }\end{array}$ & $\begin{array}{l}\text { Chloroform } \\
\text { (Ph.Chf) }\end{array}$ & $\begin{array}{l}\text { Aqueous } \\
\text { (Ph.Aq) }\end{array}$ & $\begin{array}{l}\text { Saponins } \\
\text { (Ph.Sp) }\end{array}$ & $\begin{array}{l}\text { Positive } \\
\text { Control }\end{array}$ \\
\hline Aspergillus fumigatus & +++ & ++ & +++ & +++ & +++ & ++ & +++ & +++ \\
\hline Aspergillus flavus & +++ & ++ & ++ & ++ & +++ & + & +++ & +++ \\
\hline Aspergillus niger & ++ & ++ & +++ & ++ & +++ & - & ++ & +++ \\
\hline Fusarium oxysporum & ++ & +++ & +++ & ++ & +++ & - & +++ & +++ \\
\hline
\end{tabular}

Antifungal action expressed as complete inhibition (+++), Medium inhibition (++), Mild inhibition (+) and no inhibition (-) of fungal growth. Positive Control: Amphotericin-B

Estimation of $\mathbf{L C}_{\mathbf{5 0}}$ values Median lethal concentrations $\left(\mathrm{LC}_{50}\right)$ were calculated for insecticidal, anti-Anobium and anti-Pharaoh activities, using Microsoft Excel program.

Statistical analysis All the experiments were performed in triplicate and values were expressed as means \pm SEM. One way ANOVA followed by multiple comparison Dunnett's test was used for the comparison of positive control with the test groups. The $P$ values less than 0.05 were considered as statistically significant.

\section{Results \\ Antibacterial activity Bacterial susceptibility pattern}

Majority of bacterial strains were susceptible to the tested antibiotics except amoxicillin to whom bacterial strains were resistant (Fig. 1).

Table 4 Antifungal activity of $P$. hydropiper extracts against fungal strains in disc diffusion assay

\begin{tabular}{|c|c|c|c|c|c|}
\hline \multirow[t]{2}{*}{ Samples/Fractions } & \multicolumn{5}{|c|}{ Fungal strains (DIZ in $\mathrm{mm} n=3 \mathrm{SEM}$ ) } \\
\hline & Conc. $\mu \mathrm{g} / \mathrm{ml}$ & Aspergillus fumigatus & Aspergillus flavus & Aspergillus niger & Fusarium oxysporum \\
\hline \multirow[t]{3}{*}{ Crude (Ph.Cr) } & 125 & $6.33 \pm 0.33$ & $9.00 \pm 1.15$ & $5.00 \pm 0.00$ & $9.66 \pm 1.76$ \\
\hline & 250 & $10.00 \pm 0.57$ & $16.00 \pm 0.57$ & $8.50 \pm 1.00$ & $13.33 \pm 0.88$ \\
\hline & 500 & $16.00 \pm 0.00$ & $20.33 \pm 0.88$ & $12.00 \pm 0.57$ & $18.00 \pm 0.00$ \\
\hline \multirow[t]{3}{*}{ n-Hexane (Ph.Hex) } & 125 & $6.66 \pm 0.66$ & $8.00 \pm 0.57$ & $8.00 \pm 1.15$ & $4.00 \pm 0.50$ \\
\hline & 250 & $9.33 \pm 0.88$ & $11.00 \pm 1.15$ & $11.33 \pm 0.88$ & $7.30 \pm 1.50$ \\
\hline & 500 & $11.00 \pm 1.15$ & $16.33 \pm 0.33$ & $16.00 \pm 1.00$ & $10.00 \pm 1.00$ \\
\hline \multirow[t]{3}{*}{ Ethyl acetate (Ph.EtAc) } & 125 & $14.00 \pm 0.57$ & $7.00 \pm 1.15$ & $13.66 \pm 0.66$ & $6.33 \pm 1.45$ \\
\hline & 250 & $16.00 \pm 0.33$ & $9.33 \pm 0.88$ & $17.33 \pm 2.02$ & $9.00 \pm 1.15$ \\
\hline & 500 & $21.33 \pm 0.88$ & $17.00 \pm 0.00$ & $19.66 \pm 1.76$ & $15.00 \pm 0.00$ \\
\hline \multirow[t]{3}{*}{ Butanol (Ph.Bt) } & 125 & $13.00 \pm 0.50$ & $9.00 \pm 0.50$ & $15.00 \pm 0.00$ & $12.33 \pm 0.66$ \\
\hline & 250 & $18.00 \pm 1.70$ & $14.00 \pm 1.73$ & $21.66 \pm 1.45$ & 18. $00 \pm 0.50$ \\
\hline & 500 & $22.33 \pm 1.45$ & $17.33 \pm 1.45$ & $26.00 \pm 2.30$ & $26.00 \pm 1.73$ \\
\hline \multirow[t]{3}{*}{ Chloroform (Ph.Chf) } & 125 & $13.00 \pm 1.15$ & $13.00 \pm 1.15$ & $14.33 \pm 0.88$ & $9.33 \pm 2.02$ \\
\hline & 250 & $20.33 \pm 0.88$ & $17.00 \pm 2.30$ & $19.00 \pm 1.45$ & $13.00 \pm 0.57$ \\
\hline & 500 & $26.00 \pm 1.73$ & $22.66 \pm 1.20$ & $23.00 \pm 1.15$ & $16.00 \pm 2.30$ \\
\hline \multirow[t]{3}{*}{ Aqueous (Ph.Aq) } & 125 & $10.33 \pm 0.88$ & $15.00 \pm 1.15$ & $12.00 \pm 00$ & $12.30 \pm 0.33$ \\
\hline & 250 & $13.00 \pm 1.15$ & $18.00 \pm 1.73$ & $17.00 \pm 1.45$ & $17.00 \pm 1.73$ \\
\hline & 500 & $17.00 \pm 2.30$ & $24.33 \pm 1.45$ & $21.00 \pm 0.57$ & $28.66 \pm 0.88$ \\
\hline \multirow[t]{3}{*}{ Saponins (Ph.Sp) } & 125 & $8.33 \pm 0.88$ & $11.00 \pm 0.00$ & $7.00 \pm 0.00$ & $9.00 \pm 2.30$ \\
\hline & 250 & $11.66 \pm 0.66$ & $16.00 \pm 0.50$ & $12.33 \pm 1.20$ & $12.66 \pm 1.20$ \\
\hline & 500 & $16.00 \pm 0.57$ & $18.33 \pm 1.15$ & $15.00 \pm 1.15$ & $15.00 \pm 0.00$ \\
\hline \multirow[t]{3}{*}{ Positive Control } & 125 & $19.33 \pm 0.88$ & $23.66 \pm 1.76$ & $17.00 \pm 2.88$ & $22.66 \pm 1.20$ \\
\hline & 250 & $26.00 \pm 1.15$ & $30.66 \pm 2.33$ & $22.00 \pm 2.00$ & $28.66 \pm 2.60$ \\
\hline & 500 & $33.00 \pm 0.57$ & $36.00 \pm 1.73$ & $29.00 \pm 1.15$ & $35.33 \pm 2.02$ \\
\hline N. Control & - & - - & - & - & - \\
\hline
\end{tabular}


Table 5 Minimum fungicidal concentrations (MFCs) of Polygonum hydropiper extracts and saponins

\begin{tabular}{|c|c|c|c|c|c|c|c|c|}
\hline \multirow[t]{2}{*}{ Fungal strains } & \multicolumn{8}{|c|}{ Minimum Fungicidal concentrations (MFCs) in $\mu \mathrm{g} / \mathrm{ml}$} \\
\hline & $\begin{array}{l}\text { Crude } \\
\text { (Ph.Cr) }\end{array}$ & $\begin{array}{l}\text { n-Hexane } \\
\text { (Ph.Hex) }\end{array}$ & $\begin{array}{l}\text { Ethyl acetate } \\
\text { (Ph.EtAc) }\end{array}$ & $\begin{array}{l}\text { Butanol } \\
\text { (Ph.Bt) }\end{array}$ & $\begin{array}{l}\text { Chloroform } \\
\text { (Ph.Chf) }\end{array}$ & $\begin{array}{l}\text { Aqueous } \\
\text { (Ph.Aq) }\end{array}$ & $\begin{array}{l}\text { Saponins } \\
\text { (Ph.Sp) }\end{array}$ & $\begin{array}{l}\text { Positive } \\
\text { Control }\end{array}$ \\
\hline $\begin{array}{l}\text { Aspergillus } \\
\text { fumigatus }\end{array}$ & $73.33 \pm 6.66$ & $116.66 \pm 8.33$ & $16.66 \pm 3.33$ & $33.33 \pm 6.66$ & $16.66 \pm 3.33$ & $500.00 \pm 0.00$ & $20.00 \pm 0.00$ & $8.33 \pm 1.66$ \\
\hline Aspergillus flavus & $250.00 \pm 0.00$ & $73.33 \pm 6.66$ & $60.00 \pm 0.00$ & $125.00 \pm 0.00$ & $23.33 \pm 8.81$ & $>1000$ & $53.33 \pm 6.66$ & $6.66 \pm 1.66$ \\
\hline Aspergillus niger & $100.00 \pm 0.00$ & $>1000$ & $40.00 \pm 0.00$ & $93.33 \pm 6.66$ & $125.00 \pm 0.0$ & $500.00 \pm 0.00$ & $33.33 \pm 6.66$ & $16.66 \pm 3.33$ \\
\hline $\begin{array}{l}\text { Fusarium } \\
\text { oxysporum }\end{array}$ & $86.66 \pm 6.66$ & $500.00 \pm 0.00$ & $93.33 \pm 6.66$ & $250.00 \pm 0.00$ & $46.66 \pm 6.66$ & $>1000$ & $40.00 \pm 0.00$ & $20.00 \pm 0.00$ \\
\hline
\end{tabular}

Minimum Fungicidal concentrations (MFCs) were measured at concentration range of $2.5-512 \mu \mathrm{g} / \mathrm{ml}$ after serial dilutions. Experiments were performed in triplicate. Positive Control: Amphotericin-B

\section{Disc diffusion assay}

In disc diffusion assay Ph.Chf, Ph.Hex, Ph.EtAc and Ph.Sp were found most active against E. faecalis as shown in Table 1. Most of fractions were less active against $K$. pneumonia whereas, Ph.Chf, Ph.Hex and Ph.Cr were moderately effective. Ph.Aq and Ph.Sp were most active against $E$. coli with inhibitory zones of $26.66 \pm 1.20$ and $23.00 \pm 0.00 \mathrm{~mm}$ respectively. Furthermore, Ph.Cr, Ph.Bt, Ph.EtAc and Ph.Chf were active against P. mirabilis in comparison to other fractions. Ph.Hex, Ph.Bt and Ph.Sp were most active against $S$. aureus with inhibitory zones of $26.33 \pm 0.88,23.00 \pm 1.15$ and $22.00 \pm 1.52 \mathrm{~mm}$

Table 6 Insecticidal activity of solvent extracts from Polygonum hydropiper against Tribolium castaneum and Rhyzopertha dominica

\begin{tabular}{|c|c|c|c|c|c|c|c|c|}
\hline Samples/Fractions & Total Insects & Conc. ( $\mu \mathrm{g} / \mathrm{ml})$ & $\begin{array}{l}\text { Tribolium castraneum } \\
\text { killed }\end{array}$ & $\begin{array}{l}\text { Percent } \\
\text { mortality }\end{array}$ & $\mathrm{LC}_{50} \mathrm{\mu g} / \mathrm{ml}$ & $\begin{array}{l}\text { Rhyzopertha } \\
\text { dominica Killed }\end{array}$ & $\begin{array}{l}\text { Percent } \\
\text { mortality }\end{array}$ & $\mathrm{LC}_{50} \mu \mathrm{g} / \mathrm{m}$ \\
\hline \multirow[t]{3}{*}{ Crude (Ph.Cr) } & 30 & 125 & $12.00 \pm 0.57$ & $40.00^{* * *}$ & 255 & $19.00 \pm 0.00$ & $63.33^{* *}$ & 25 \\
\hline & 30 & 250 & $14.33 \pm 0.66$ & $47.76^{* * *}$ & & $21.00 \pm 1.15$ & $70.00^{* *}$ & \\
\hline & 30 & 500 & $18.33 \pm 0.33$ & $61.10^{* *}$ & & $22.67 \pm 0.88$ & $75.56^{* *}$ & \\
\hline \multirow[t]{3}{*}{ n-Hexane (Ph.Hex) } & 30 & 125 & $12.66 \pm 0.33$ & $42.20^{* * *}$ & 360 & $13.00 \pm 0.57$ & $43.33^{* * *}$ & 190 \\
\hline & 30 & 250 & $15.66 \pm 0.66$ & $62.20^{* *}$ & & $16.66 \pm 0.33$ & $55.53^{* * *}$ & \\
\hline & 30 & 500 & $18.67 \pm 0.33$ & $62.23^{* *}$ & & $19.00 \pm 0.58$ & $63.33^{* *}$ & \\
\hline \multirow[t]{3}{*}{ Ethyl acetate (Ph.EtAc) } & 30 & 125 & $17.66 \pm 0.66$ & $58.86^{* * *}$ & 80 & $15.33 \pm 0.88$ & $51.10^{* * *}$ & 110 \\
\hline & 30 & 250 & $20.00 \pm 0.57$ & $66.66^{* *}$ & & $23.00 \pm 1.15$ & $76.66^{* *}$ & \\
\hline & 30 & 500 & $24.00 \pm 0.57$ & $80.00^{*}$ & & $26.33 \pm 0.33$ & $87.76^{*}$ & \\
\hline \multirow[t]{3}{*}{ Butanol (Ph.Bt) } & 30 & 125 & $21.33 \pm 0.33$ & $71.10^{* *}$ & 20 & $19.66 \pm 1.88$ & $65.53^{* *}$ & 57 \\
\hline & 30 & 250 & $24.00 \pm 0.57$ & $80.00^{*}$ & & $25.00 \pm 1.15$ & $83.33^{*}$ & \\
\hline & 30 & 500 & $26.67 \pm 0.89$ & $88.90^{*}$ & & $28.00 \pm 0.00$ & 93.33 & \\
\hline \multirow[t]{3}{*}{ Chloroform (Ph.Chf) } & 30 & 125 & $4.66 \pm 0.88$ & $15.53^{* * *}$ & $>500$ & $5.00 \pm 0.57$ & $16.55^{* * *}$ & $>500$ \\
\hline & 30 & 250 & $6.33 \pm 0.33$ & $21.10^{* * *}$ & & $9.00 \pm 1.15$ & $30.00^{* * *}$ & \\
\hline & 30 & 500 & $8.67 \pm 0.89$ & $28.90^{* * *}$ & & $11.67 \pm 0.88$ & $38.90^{* * *}$ & \\
\hline \multirow[t]{3}{*}{ Aqueous (Ph.Aq) } & 30 & 125 & $14.00 \pm 0.00$ & $46.66^{* * *}$ & 130 & $9.66 \pm 0.88$ & $32.20 * * *$ & 300 \\
\hline & 30 & 250 & $21.00 \pm 1.15$ & $70.00^{* *}$ & & $14.00 \pm 0.57$ & $46.66^{* * *}$ & \\
\hline & 30 & 500 & $23.33 \pm 1.21$ & $77.77^{* *}$ & & $18.33 \pm 0.33$ & $61.10^{* *}$ & \\
\hline \multirow[t]{3}{*}{ (Saponins) Ph.Sp } & 30 & 125 & $15.33 \pm 0.88$ & $51.10^{* * *}$ & 110 & $21.33 \pm 1.20$ & $71.10^{* *}$ & 28 \\
\hline & 30 & 250 & $22.00 \pm 0.00$ & $73.33^{* *}$ & & $24.00 \pm 0.00$ & $80.00^{*}$ & \\
\hline & 30 & 500 & $25.00 \pm 0.00$ & $83.33^{*}$ & & $27.00 \pm 0.00$ & 90.00 & \\
\hline \multirow[t]{3}{*}{ Positive Control } & 30 & 125 & $24.00 \pm 0.00$ & 80.00 & 15 & $24.66 \pm 0.33$ & 82.20 & 9 \\
\hline & 30 & 250 & $27.66 \pm 0.88$ & 92.20 & & $27.33 \pm 0.88$ & 91.10 & \\
\hline & 30 & 500 & $30.00 \pm 0.00$ & 100.00 & & $30.00 \pm 0.00$ & 100.00 & \\
\hline Negative Control & 30 & - & $0 \pm 0.00$ & $0 \pm 0.00$ & - & $0 \pm 0.00$ & $0 \pm 0.00$ & \\
\hline
\end{tabular}

Data was represented as mean \pm SEM $(n=3)$ of three independent experimental readings. Positive control: Permethrin. Negative Control: solvents used for dissolution. Values significantly different when compared to standard drug *: $0.05,{ }^{* *}: 0.01$ and ***: 0.001 at $90 \%$ confidence interval 
respectively. Ph.Chf was most active against S. typhi and $P$. aeruginosa.

\section{Minimum Inhibitory Concentrations (MICs)}

Results of MICs are summarized in Table 2. Ph.Chf and Ph.Sp were most active against bacterial strains. The MICs of Ph.Chf against E. faecalis, K. pneumonia, E. coli, P. mirabilis, S. aureus, S. typhi and P. aeruginosa were $32.00 \pm$ $0.00,13.33 \pm 2.66,10.66 \pm 2.66,5.33 \pm 1.33,64.00 \pm 0.00$, $8.66 \pm 0.66$ and $10.66 \pm 2.66 \mu \mathrm{g} / \mathrm{ml}$ respectively. Ph.Sp has exhibited lower MIC values against $E$. faecalis (10.66 \pm 2.66), K. pneumonia (32.00 \pm 0.00$)$, E. coli $(26.66 \pm 5.33), P$. mirabilis $(6.66 \pm 1.33)$, S. aureus $(128.00 \pm 0.00)$, S. typhi $(53.33 \pm 10.66)$ and $P$. aeruginosa $(6.66 \pm 1.33) \mu \mathrm{g} / \mathrm{ml}$. Results of these fractions were comparable to positive control.

\section{Antifungal activity}

Preliminary antifungal assay Results were expressed in the form of complete growth inhibition $(+++)$, moderate inhibition $(++)$ and partial $(+)$ inhibition. All fractions showed antifungal activities. Ph.Chf was found most effective, causing complete inhibition of visible fungal growth against all tested strains (Table 3). Among other fractions, Ph.Sp, Ph.Cr and Ph.Bt showed prominent antifungal activity. Antifungal action of other fractions was mild to moderate.

Antifungal disc diffusion assay Results of antifugal activity are given in Table 4. Ph.Chf, Ph.Bt and Ph.EtAc were most active against $A$. fumigatus scoring inhibitory zones of $26.00 \pm 1.73,22.33 \pm 1.45$ and $21.33 \pm 0.88 \mathrm{~mm}$ respectively at $500 \mu \mathrm{g} / \mathrm{ml}$. Ph.Chf and Ph.Aq exhibited inhibitory zones of $22.66 \pm 1.20$ and $24.33 \pm 1.45 \mathrm{~mm}$ respectively against $A$. flavus at highest tested concentration. Further, Ph.Bt, Ph.Chf and Ph.Aq showed highest activity against $A$. niger. In activity against $F$. oxysporum, $\mathrm{Ph} . \mathrm{Bt}$ and $\mathrm{Ph} . \mathrm{Aq}$ were found more effective in comparison to other fractions. Rest of the fractions showed moderate activity against the fungal strains.

Table 7 Larvicaidal activity of Polygonum hydropiper extracts against Anobium punctatum

\begin{tabular}{|c|c|c|c|c|c|c|}
\hline Samples & Dose $(\mathrm{mg} / \mathrm{ml})$ & Total treated & No. Repeated & Average lethality & \%lethality mean \pm SEM & $\mathrm{LC}_{50}(\mathrm{mg} / \mathrm{ml})$ \\
\hline \multirow[t]{3}{*}{ Crude (Ph.Cr) } & 12.5 & 25 & 3 & $17.66 \pm 0.66$ & 70.64 & 0.93 \\
\hline & 25 & 25 & & $18.66 \pm 0.33$ & 74.64 & \\
\hline & 50 & 25 & & $20.00 \pm 0.57$ & 80.00 & \\
\hline \multirow[t]{3}{*}{ n-Hexane (Ph.Hex) } & 12.5 & 25 & 3 & $8.66 \pm 0.88$ & 34.64 & 27.32 \\
\hline & 25 & 25 & & $12.33 \pm 0.88$ & 49.32 & \\
\hline & 50 & 25 & & $14.66 \pm 1.20$ & 58.64 & \\
\hline \multirow[t]{3}{*}{ Chloroform (Ph.Chf) } & 12.5 & 25 & 3 & $19.33 \pm 0.88$ & 77.32 & 1.16 \\
\hline & 25 & 25 & & $22.33 \pm 1.20$ & 89.32 & \\
\hline & 50 & 25 & & $23.33 \pm 0.66$ & 93.32 & \\
\hline \multirow[t]{3}{*}{ Ethyl acetate (Ph.EtAc) } & 12.5 & 25 & 3 & $15.66 \pm 1.33$ & 62.64 & 6.35 \\
\hline & 25 & 25 & & $17.66 \pm 2.33$ & 70.64 & \\
\hline & 50 & 25 & & $21.00 \pm 1.15$ & 84.00 & \\
\hline \multirow[t]{3}{*}{ Aqueous (Ph.Aq) } & 12.5 & 25 & 3 & $9.66 \pm 1.20$ & 38.64 & 53.24 \\
\hline & 25 & 25 & & $11.00 \pm 1.15$ & 44.00 & \\
\hline & 50 & 25 & & $12.33 \pm 0.88$ & 49.32 & \\
\hline \multirow[t]{3}{*}{ Butanol (Ph.Bt) } & 12.5 & 25 & 3 & $16.66 \pm 0.33$ & 66.64 & 2.28 \\
\hline & 25 & 25 & & $17.33 \pm 0.88$ & 69.32 & \\
\hline & 50 & 25 & & $19.66 \pm 2.33$ & 78.64 & \\
\hline \multirow[t]{3}{*}{ (Saponins) Ph.Sp } & 12.5 & 25 & 3 & $23.66 \pm 0.33$ & 94.64 & $<0.01$ \\
\hline & 25 & 25 & & $24.00 \pm 0.00$ & 96.00 & \\
\hline & 50 & 25 & & $25.00 \pm 0.00$ & 100.00 & \\
\hline \multirow[t]{3}{*}{ Positive Control } & 12.5 & 25 & 3 & $25.00 \pm 0.00$ & 100.0 & $<0.01$ \\
\hline & 25 & 25 & & $25.00 \pm 0.00$ & 100.0 & \\
\hline & 50 & 25 & & $25.00 \pm 0.00$ & 100.0 & \\
\hline Negative Control & - & 25 & 3 & 0.00 & 0.00 & 0.00 \\
\hline
\end{tabular}

Each value represent Mean \pm SEM of three independent experimental readings. Results were expressed as $\% \mathrm{mortality}$ and $\mathrm{LC}_{50}(\mathrm{mg} / \mathrm{ml})$. Negative Control: Distilled Water, Positive Control: permethrin 
Minimum Fungicidal Concentrations (MFCs) In MFCs determination assay, Ph.Chf was highly effective against A. fumigatus, A. flavus, $A$. niger and $F$. oxysporum exhbiting MFCs of $16.66 \pm 3.33,23.33 \pm 8.81,125.00 \pm$ 0.00 and $46.66 \pm 6.66 \mu \mathrm{g} / \mathrm{ml}$ respectively (Table 5 ). Ph.Sp, Ph.EtAc and Ph.Bt and were most effective against $A$. fumigatus showed MFCs of $20.00 \pm 0.00,16.66 \pm 3.33$ and $33.33 \pm 6.66 \mu \mathrm{g} / \mathrm{ml}$. Ph.Aq was least effective against the tested fungi exhibiting MFCs of $>1000 \mu \mathrm{g} / \mathrm{ml}$ against $A$. flavus and $F$. oxysporum.

Insecticidal study against $T$. castaneum and $R$. dominica Results of insecticidal activity against $T$. castaneum and $R$. dominica are given in Table 6. Among different fractions, Ph.EtAc, Ph.Sp, Ph.Chf and Ph.Bt were most active exhibiting $\mathrm{LC}_{50}$ of $20,110,80$ and $130 \mu \mathrm{g} / \mathrm{ml}$ respectively. Moreover, insecticidal action of Ph.EtAc, Ph.Sp, Ph.Chf and Ph.Cr were most prominent against $R$. dominica with $\mathrm{LC}_{50}$ of $57,28,110$ and $25 \mu \mathrm{g} /$ $\mathrm{ml}$ respectively.
Anti- anobium activity Results of larvicidal activity against A. punctatum are given in Table 7 . Ph.Sp was found most active causing 94.64, 96.00 and $100.00 \%$ lethality of tested larvae at $12.5,25$ and $50 \mathrm{mg} / \mathrm{ml} \mathrm{re-}$ spectively. Saponins activity was comparable with standard drug permethrin, causing $100 \%$ death of all larvae with $\mathrm{LC}_{50}$ of $<0.01$. Among other fractions, $\mathrm{Ph} . \mathrm{Chf}$, Ph.EtAc and Ph.Cr were most active causing 93.32, 84.00 and $80.00 \%$ lethality against A. punctatum with $\mathrm{LC}_{50} 1.16,6.35$ and $0.93 \mathrm{mg} / \mathrm{ml}$ respectively at $50 \mathrm{mg} / \mathrm{ml}$. Ph.Hex, Ph.Bt and PhAq showed moderate activity.

Anti-pharaoh activity In Anti-Pharaoh investigations Ph.Sp was found most active against the tested ants showing $93.30,100.00$ and $100.00 \%$ lethality at concentrations of $12.5,25$ and $50 \mathrm{mg} / \mathrm{ml}$ respectively with $\mathrm{LC}_{50}$ of $<0.01 \mathrm{mg} / \mathrm{ml}$. Activity of Ph.Sp was comparable with standard drug at the same tested concentration. Ph.Chf was also equally effective, causing $83.30,86.65$ and

Table 8 Anti-Pharaoh investigations of $P$. hydropiper extracts and saponins

\begin{tabular}{|c|c|c|c|c|c|c|}
\hline Samples & Dose $(\mathrm{mg} / \mathrm{ml})$ & Total treated & No. Repeated & Average lethality & Percent lethality & $\mathrm{LC}_{50}(\mathrm{mg} / \mathrm{ml})$ \\
\hline \multirow[t]{3}{*}{ Crude (Ph.Cr) } & 12.5 & 20 & 3 & $8.66 \pm 0.66$ & 43.30 & 33.54 \\
\hline & 25 & 20 & & $9.00 \pm 1.15$ & 45.00 & \\
\hline & 50 & 20 & & $12.66 \pm 2.33$ & 63.30 & \\
\hline \multirow[t]{3}{*}{ n-Hexane (Ph.Hex) } & 12.5 & 20 & 3 & $5.00 \pm 0.57$ & 25.00 & 54.82 \\
\hline & 25 & 20 & & $7.66 \pm 0.88$ & 38.30 & \\
\hline & 50 & 20 & & $9.33 \pm 0.88$ & 46.65 & \\
\hline \multirow[t]{3}{*}{ Chloroform (Ph.Chf) } & 12.5 & 20 & 3 & $16.66 \pm 1.33$ & 83.30 & $<0.01$ \\
\hline & 25 & 20 & & $17.33 \pm 0.66$ & 86.65 & \\
\hline & 50 & 20 & & $19.33 \pm 0.88$ & 96.65 & \\
\hline \multirow[t]{3}{*}{ Ethyl acetate (Ph.EtAc) } & 12.5 & 20 & 3 & $12.00 \pm 0.00$ & 60.00 & 5.91 \\
\hline & 25 & 20 & & $14.00 \pm 1.15$ & 70.00 & \\
\hline & 50 & 20 & & $15.66 \pm 0.33$ & 78.30 & \\
\hline \multirow[t]{3}{*}{ Aqueous (Ph.Aq) } & 12.5 & 20 & 3 & $9.66 \pm 1.20$ & 48.30 & 17.17 \\
\hline & 25 & 20 & & $10.66 \pm 1.33$ & 53.30 & \\
\hline & 50 & 20 & & $12.33 \pm 0.88$ & 61.65 & \\
\hline \multirow[t]{3}{*}{ Butanol (Ph.Bt) } & 12.5 & 20 & 3 & $12.00 \pm 1.15$ & 60.00 & 6.13 \\
\hline & 25 & 20 & & $13.66 \pm 0.88$ & 68.30 & \\
\hline & 50 & 20 & & $15.66 \pm 0.66$ & 78.30 & \\
\hline \multirow[t]{3}{*}{ (Saponins) Ph.Sp } & 12.5 & 20 & 3 & $18.66 \pm 0.33$ & 93.30 & $<0.01$ \\
\hline & 25 & 20 & & $20.00 \pm 0.00$ & 100.00 & \\
\hline & 50 & 20 & & $20.00 \pm 0.00$ & 100.00 & \\
\hline \multirow[t]{3}{*}{ Positive Control } & 12.5 & 25 & 3 & $25.00 \pm 0.00$ & 100.0 & $<0.01$ \\
\hline & 25 & 25 & & $25.00 \pm 0.00$ & 100.0 & \\
\hline & 50 & 25 & & $25.00 \pm 0.00$ & 100.0 & \\
\hline Negative Control & - & 25 & 3 & 0.00 & 0.00 & 0.00 \\
\hline
\end{tabular}

Negative Control: Distilled Water; Positive Control: Permethrin 
$96.65 \%$ death of $M$. pharaonis at concentrations of 12.5 , 25 and $50 \mathrm{mg} / \mathrm{ml}$ with $\mathrm{LC}_{50}$ of $<0.01 \mathrm{mg} / \mathrm{ml}$. All other fractions showed mild to moderate activity as shown in Table 8.

GC-MS analysis In GC-MS analysis of Ph.Cr, 124 compounds were identified (Additional file 1: Table S1). Overall, nine compounds were found dominant including 2,3-dihydro benzofuran, humulene oxide, caryophyllene epoxide, $2 \mathrm{H}$-cyclopropa benzofuran, neophytadiene 7,11,15 trimethyl,3-methylene-1-hexadecene, 3,7,11,15tetramethyl-2-hexadecen-1-ol, 3,7,11,15-Tetramethyl-2hexadecen-1, 9,12-octadecadienoic acid methyl ester, $(E, E)$-methyl linolelaidate and 11,14,17-eicosatrienoic acid, methyl ester with concentrations of $7.89,3.54,3.68$, $3.18,25.2,6.44,10.71,3.41$ and $5.84 \%$ respectively (Fig. 2).

Identification of bioactive compounds Several bioactive antibacterial, antifungal and insecticidal compounds were identified in GC, GC-MS analysis of Ph.Cr (Fig. 3). These compounds include 4-methyloxazole, succinimide, pyrocatechol, caryophyllene, vanillic acid, farnesol, Myristic acid, arachidic acid methyl ester and capsaicin.

\section{Discussion}

Infectious diseases are among the leading health problems, accounting for $41 \%$ of global disease Burdon [33]. The development of resistance by multidrug resistant (MDR) pathogens is a major problem in the chemotherapeutic management of infectious diseases. Due to the development of resistance against synthetic drugs, researchers are focusing on natural products to find novel antibacterial, antifungal and anti-parasitic agents [34-36]. Plant based remedies are rich sources of safe and effective drugs and are used throughout the history of mankind in crude form as well as in the form of pure isolated compounds [37]. A variety of medicinal plants have been indicated for the treatment of infectious diseases in different phytotherapy manuals due to their reduced toxicity, fewer side effects and frequent availability. Different studies have been conducted on the antimicrobial potentials of plants and their efficacy has been reported in the treatment of urinary tract infections (UTIs), respiratory, cutaneous infections, neurological and gastrointestinal disorders $[38,39]$.

In our current investigations, $P$. hydropiper extracts and saponins revealed a broad spectrum of activity against pathogenic bacterial and fungal strains. Antibacterial and antifungal potential of these fractions can be attributed to their phenolic contents and the presence of different

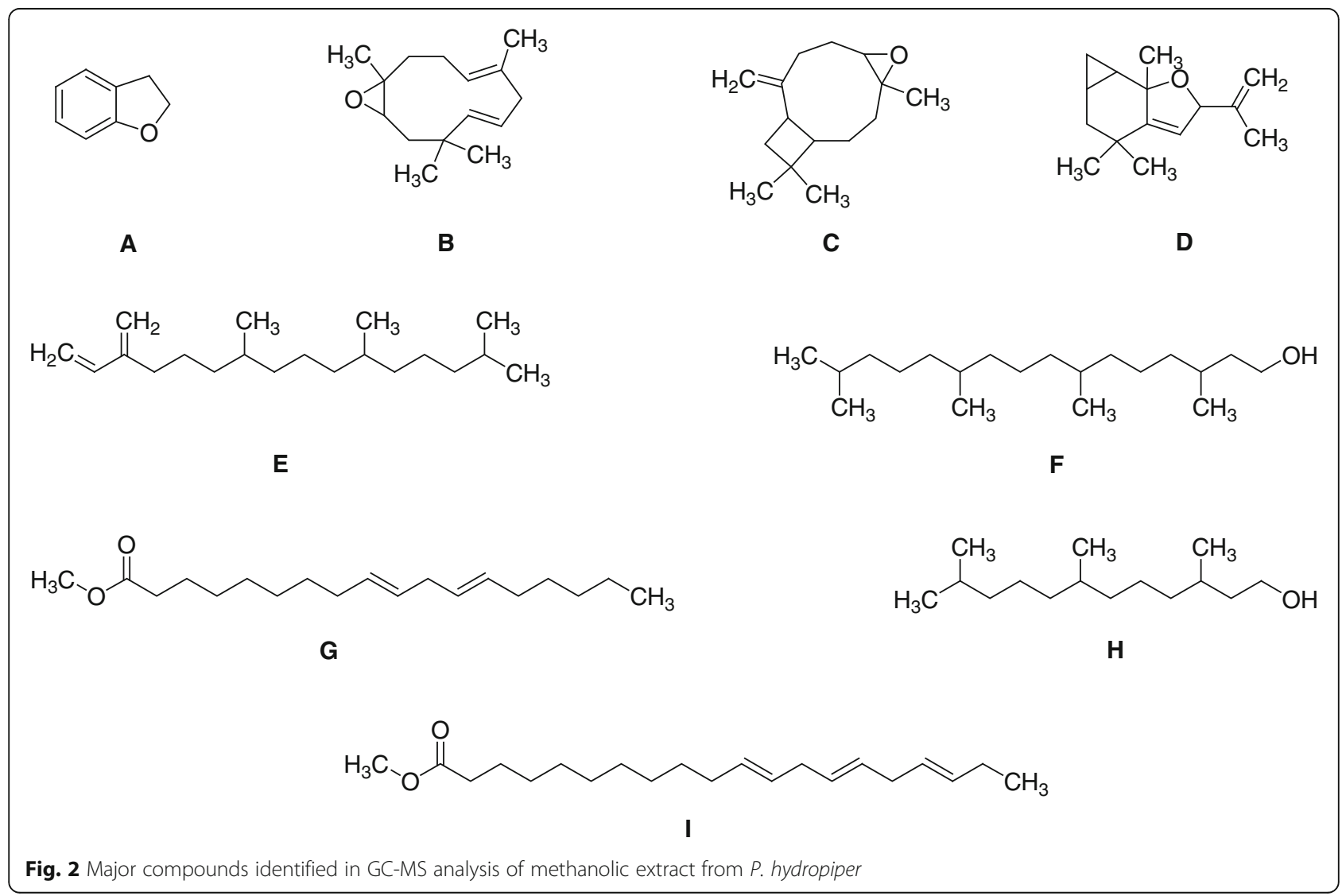




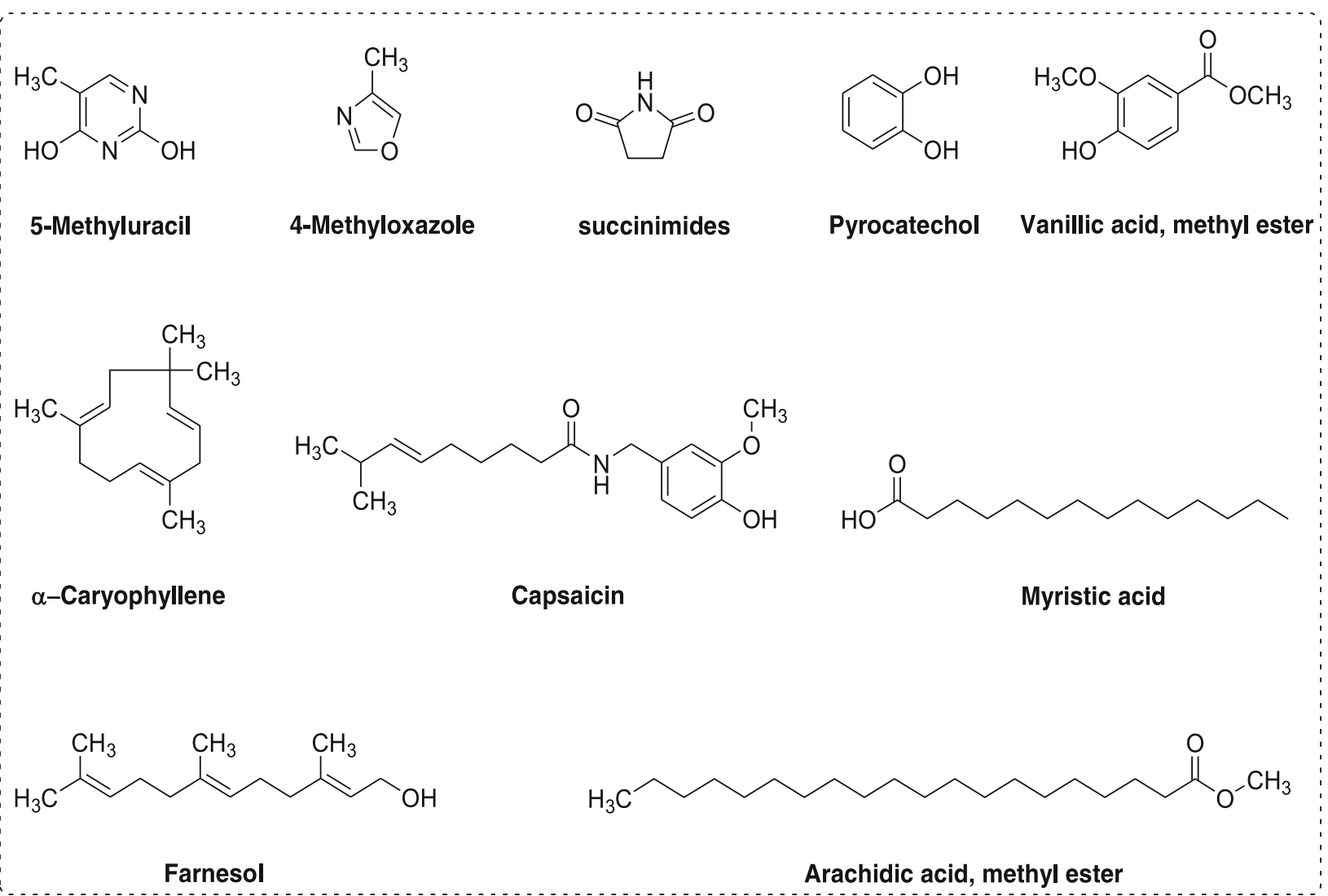

Fig. 3 Bioactive compounds identified in GC-MS analysis of methanolic extract from P. hydropiper

pharmacologically active compounds. As indicated by their MICs and DIZ values, Ph.Chf, Ph.Hex and Ph.Sp showed most prominent activity against the tested bacterial strains whereas, Ph.Aq was least active. DIZ and MICs of these fractions correlated well with each other in relation to antibacterial activities. Broadly, samples were more active against Gram negative strains in comparison to Gram positive. In antifungal assays, Ph.EtAc, Ph.Bt, Ph.Chf and Ph.Sp exhibited prominent activity against fungal strains whereas, Ph.Aq was found least effective in MFCs assay (Tables 1, 2, 3, 4 and 5). Majority of fungal stains were inhibited at MFCs range of $16.66-1000 \mu \mathrm{g} / \mathrm{ml}$.

Several bioactive compounds were identified in the GC-MS spectra of $P$. hydropiper including thymin, 4methyloxazole, succinimide, vanillic acid, caryophyllene, farnesol, capsaicin, myristic acid, arachidic acid, methyl palmitate etc (Additional file 1: Table S1). Thymin, 4methyloxazole, succinimide, pyrocatechol and caryophyllene has been previously reported for antibacterial and antifungal potential [40-45]. Furthermore, phenolic acid, farnesol, myristic acid, Arachidic acid methyl ester and capsaicin has been demonstrated against pathogenic bacteria and fungi [46-52]. The antimicrobial action of $P$. hydropiper can be attributed to the presence of these bioactive compounds.

Higher plants are good sources of novel compounds that can be used to develop environment friendly insecticidal drugs [53]. Insecticidal potentials of several plants against different insect pests has been reported by several groups [54]. A possibly interesting group of molecules is the saponins, a class of steroidal or triterpenoidal secondary plant metabolites having divergent biological activities [55]. T. castaneum and $R$. dominica are considered major pests of stored grains and food products. Annual post-harvest losses resulting from insect infestations, microbial deterioration and others factors is estimated to be $10-25 \%$ worldwide [56]. Control of these insects relies heavily on the utilization of synthetic insecticides and fumigants. However, their extensive use has led to some stern problems including development of insect strains resistant to insecticides, deposition of toxic residues on stored grain, toxicity to users and high costs of application [57]. There is critical need to develop safe and cost-effective alternatives which are convenient for user and environment friendly.

In our current insecticidal study, Ph.EtAc, Ph.Sp and Ph.Chf were most effective against $T$. castaneum with $\mathrm{LC}_{50}$ 
of 20,110 and $80 \mu \mathrm{g} / \mathrm{ml}$ respectively which was comparable with standard drug permethrin. Similarly, Ph.EtAc, Ph.Sp and Ph.Chf were also most effective against $R$. dominica with $\mathrm{LC}_{50}$ of 57,28 and $110 \mu \mathrm{g} / \mathrm{ml}$ respectively. Insecticidal potential of these fractions was comparable with the positive control at the same tested concentration. In larvicidal activity against $A$. punctatum, we observed that Ph.Sp were highly active with $\mathrm{LC}_{50}$ of $<0.01 \mathrm{mg} / \mathrm{ml}$ which was comparable with standard drug permethrin at the same tested concentration. Larvicidal potentials of $\mathrm{Ph} . \mathrm{Chf}$, Ph.EtAc and Ph.Cr were also prominent with $\mathrm{LC}_{50}$ of 1.16, 6.35 and $0.93 \mathrm{mg} / \mathrm{ml}$ respectively. In anti-Pharaoh assay, again $\mathrm{Ph} . \mathrm{Sp}$ and $\mathrm{Ph} . \mathrm{Chf}$ were most potent fractions showing $\mathrm{LC}_{50}$ of $<0.01 \mathrm{mg} / \mathrm{ml}$. Our current finding support previous insecticidal reports on saponins, and suggests that the saponins from $P$. hydropiper can be a cost-effective source of insecticidal compounds.

Other fractions with promising results can be subjected to activity guided isolation to obtain novel and more effective drugs against infectious diseases, insects and pests. We identified several insecticidal compounds in GC-MS analysis. Among these, the insecticidal activity of farnesol has also been reported [58]. Methyl palmitate and myristic acid also possess insecticidal properties [59, 60].

\section{Conclusions}

Results of the current study indicate that $P$. hydropiper possess broad spectrum antimicrobial activity and signifies its potential as a source of therapeutic agent against bacterial and fungal infections. Further studies, regarding isolation and purification of novel bioactive component, can depict the precise potentials of the plant to restrain pathogenic microbes since the purified compounds may have even more efficacy with respect to inhibition of microbes. Our findings regarding antimicrobial and insecticidal activities, exhibited by extracts and saponins may offer scientific justification for the ethnomedicinal uses of the plant.

\section{Additional file}

Additional file 1: Table S1. Result of GC, GC-MS analysis for the identification of compounds in Ph.Cr of P.hydropiper. (DOCX $25 \mathrm{~kb}$ )

\footnotetext{
Abbreviations

A. flavus: Aspergillus flavus; A. fumigatus: Aspergillus fumigatus; A. niger: Aspergillus niger; A. punctatum: Anobium punctatum; CLSI: Clinical and laboratory standard institute; DIZ: Diameter of Inhibitory zone; E. faecalis: Enterococcus faecalis; E.coli: Escherichia col; F. oxysporum: Fusarium oxysporum; GC-MS: Gas chromatography-mass spectrometry; K. pneumonia: Klebsella pneumonia; $M$. pharaonis: Monomorium pharaonis; MDR: Multidrug resistant; MFCs: Minimum fungicidal concentration; MICs: Minimum inhibitory concentration; $P$ aeruginosa: Pseudomonas aeruginosa; P. hydropiper: Polygonum hydropiper; P. mirabilis: Proteus mirabilis; Ph.Aq: Aqueous; Ph.Bt: n-Butanol; Ph.Chf: Chloroform; Ph.Cr: Crude extract; Ph.EtAc: Ethyl acetate; Ph.Hex: n-hexane; Ph.Sp: Crude saponins; R. dominica: Rhyzopertha dominica; S. aureus: Staphylococcus aureu; S.
}

typhi: Salmonella typhi; SDA: Sabouraud dextrose Agar; T. castaneum: Tribolium castaneum

\section{Acknowledgements}

We are grateful to Microbiology Laboratory Quaid-i-Azam University Islamabad, Pakistan for providing bacterial and fungal strains for research purpose. The authors are also grateful to Saeed Ahmad, Assistant Professor of Zoology, University of Malakand for the identifications of the insects and Department of Pharmacy, University of Malakand, Khyber Pakhtoonkhwa, Pakistan, for providing laboratory facilities to conduct this research. We are also grateful to Mr. Muhammad Aziz, Department of English for language editing of the manuscript.

\section{Funding}

This research has received no specific grant from any funding agency in the public, commercial, or not for-profit sectors.

\section{Availability of data and materials}

The data presented in this manuscript belong to research work of Muhammad Ayaz and has not been deposited in any repository yet. However, the materials are available to the researchers upon request.

\section{Authors' contributions}

MA carried out experimental work, data collection, evaluation, literature search and manuscript preparation. MJ and FU supervised research work, helped in study design and drafted the final version of the manuscript. SA, AZ helped in insects' collection and insecticidal experiments. AS, WA and $\mathrm{MO}$ helped in interpretation, statistical analysis of the data and refined the manuscript for publication. All authors read and approved the final manuscript for publication.

\section{Competing interests}

The authors declare that they have no competing interests.

\section{Consent for publication}

Not applicable in this section.

\section{Ethics approval and consent to participate}

Not applicable in this Manuscript.

\section{Author details}

${ }^{1}$ Department of Pharmacy, University of Malakand, Khyber Pakhtoonkhwa (KPK) 18000, Pakistan. ${ }^{2}$ Cancer Biology Lab (MOSEL), Department of Biotechnology, Quaid-i-Azam University, Islamabad, Pakistan.

Received: 16 May 2016 Accepted: 30 November 2016 Published online: 05 December 2016

\section{References}

1. Alanis A. Resistance to antibiotics: are we in the post-antibiotic era? Arch Med Res. 2005;36:697-705.

2. Ayaz M, Subhan F, Ahmed J, Khan A-u, Ullah F, Ullah I, Ali G, Hussain S. Sertraline enhances the activity of antimicrobial agents against pathogens of clinical relevance. J Biol Res Thessaloniki. 2015;22(1):4.

3. Sharma D, Narasimhan B, Kumar P, Judge V, Narang N, De Clerq E, Balzarini J. Synthesis, antimicrobial and antiviral evaluation of substituted imidazole derivatives. Eur J Med Chem. 2009;44:2347-53.

4. Livermore DM. Discovery research: the scientific challenge of finding new antibiotics. J Antimicrob Chemother. 2011;66:1941-4.

5. Ayaz M, Subhan F, Ahmed J, Khan A-u, Ullah F, Sadiq A, Syed N-I-H, Ullah I, Hussain S. Citalopram and venlafaxine differentially augments antimicrobial properties of antibiotics. Acta Polon Pharm Drug Res. 2015;72(6):1269-78.

6. Pelczar M, Chan E, Krieg N. Microbiology today. New York: McGraw Hill; 2006. p. 298-433.

7. Bhaskarwar B, Itankar P, Fuluke A. Evaluation of antimicrobial activity of medicinal plant Jatropha podagrica (Hook).Rom. Biotechnol Lett. 2008;13(5):3873-7.

8. World Health Organization. The promotion and development of traditional medicine. Geneva: WHO; 1978. p. 622.

9. Farnsworth N, Akerele O, Bingel A, Soejarto D, Guo Z. Medicinal plants in therapy. Bull World Health Organ. 1985;63(6):965-81. 
10. Peeyush K, Mishra S, Satya A. Insecticidal properties of Mentha species: a review. Ind Crops Prod. 2011;34(1):802-17.

11. Rajashekar Y, Bakthavatsalam N, Shivanandappa T. Botanicals as grain protectants. Psyche. 2012;2012:13. Article ID 646740. doi:10.1155/2012/646740.

12. Campbell J. Attraction of walking tribolium castaneum adults to traps. J Stored Prod Res. 2012;51(1):11-22.

13. Pugazhvendan S, Ross P, Elumalai K. Insec-ticidal and repellent activities of plants oil against stored grain pest, Tribolium castaneum (Herbst) (Coleoptera:Tenebrionidae). Asian Pacif J Trop Med. 2012;5:S412-5.

14. Ebeling W. Urban entomology. Riverside: University of California Press; 1978.

15. Sharma R. Medicinal plants of India-an encyclopedia. Delhi: Daya Publishing House; 2003. p. 46-7.

16. Chevallier A. The encyclopedia of medicinal plants. London: Dorling Kindersley; 1996. p. 185. ISBN 9-780751-303148.

17. Ayaz M, Junaid M, Ahmed J, Ullah F, Sadiq A, Ahmad S, Imran M. Phenolic contents, antioxidant and anticholinesterase potentials of crude extract, subsequent fractions and crude saponins from Polygonum hydropiper $\mathrm{L}$. BMC Complement Altern Med. 2014;14:145.

18. Ayaz M, Junaid M, Subhan F, Ullah F, Sadiq A, Ahmad S, Imran M, Kamal Z, Hussain S, Shah S. Heavy metals analysis, phytochemical, phytotoxic and anthelmintic investigations of crude methanolic extract, subsequent fractions and crude saponins from Polygonum hydropiper L. BMC Complement Altern Med. 2014;14:465.

19. Ayaz M, Junaid M, Ullah F, Sadiq A, Khan MA, Ahmad W, Shah MR, Imran M, Ahmad S. Comparative chemical profiling, cholinesterase inhibitions and anti-radicals properties of essential oils from Polygonum hydropiper $L$ : A Preliminary anti-Alzheimer's study. Lipids Health Dis. 2015;14(1):141.

20. Ayaz M, Junaid M, Ullah F, Sadiq A, Subhan F, Khan MA, Ahmad W, Ali G, Imran M, Ahmad S. Molecularly characterized solvent extracts and saponins from Polygonum hydropiper $L$ show high anti-angiogenic, anti-tumor, brine shrimp and fibroblast NIH/3T3 cell line cytotoxicity. Front Pharmacol. 2016;7:74,

21. Zeb A, Sadiq A, Ullah F, Ahmad S, Ayaz M. Phytochemical and toxicological investigations of crude methanolic extracts, subsequent fractions and crude saponins of Isodon rugosus. Biol Res. 2014;47:57.

22. Ullah F, Ayaz M, Sadiq A, Hussain A, Ahmad S, Imran M, Zeb A. Phenolic, flavonoid contents, anticholinesterase and antioxidant evaluation of Iris germanica var; florentina. Nat Prod Res. 2016;30(12):1440-4.

23. Ahmad S, Ullah F, Sadiq A, Ayaz M, Imran M, Ali I, Zeb A, Ullah F, Shah M. Chemical composition, antioxidant and anticholinesterase potentials of essential oil of Rumex hastatus D. Don collected from the North West of Pakistan. BMC Complement Altern Med. 2016;16:29.

24. Barrow Gl, Feltham RKA. In Cowan and Steel's Manual of the Identification of Medical Bacteria. Cambridge: Cambridge University Press; 1993. p. 50-9. 159-164.

25. Cruickshank R, Duguid JP, Marmion BP, Swain RHA. In medical microbiology. London: Churchill Livingstone; 1989. p. 201-8.

26. Standards NCfCL. Methods fordilution in antimicrobial susceptibility tests. Villanova: NCCLS; 1993. Approved standard M2-A5.

27. Shah SM, Ayaz M, Khan A-u, Ullah F, Farhan, Shah A-u, Iqbal H, Hussain S. 1,1-Diphenyl,2-picrylhydrazyl free radical scavenging, bactericidal, fungicidal and leishmanicidal properties of Teucrium stocksianum. Toxicol Ind Health. 2015;31(11):1037-43.

28. Dash S, Nath LK, Bhise S. Antioxidant and antimicrobial activities of Heracleum nepalense D. Don root. Trop J Pharm Res. 2005;4:341-7.

29. Khan A, Rahman M, Islam S. Antibacterial, antifungal and cytotoxic activities of Tuberous Roots of Amorphophallus campanulatus. Turk J Biol. 2007;31:167-72.

30. Atta-ur-Rahman, Choudhary M, William J. Bioassay techniques for drug development. Amsterdam: Harwood Academic Publishers; 2001. 67-68,

31. Salihah Z, Khatoon R, Khan A, Alamzeb SA. A termite trap, NIFATERMAP, for capturing large number of field population of Heterotermes indicola. Proc Pakistan Cong Zool. 1993;1993:395-400.

32. Ahn Y, Kim G, Cho K. Bioassay system for insecticidal compounds. In: Proceedings of the third symposium on the biochemical methodology for the research and development of the bioactive substances, held at Seoul, Republic of Korea: 1995. 1995. p. 495-506.

33. Ullah F, Salman Akbar M, Jawad A, Farman U, Syed Majid S, Muhammad A Sajid $H$, Khatoon L. Investigation of the genetic basis of tetracycline resistance in Staphylococcus aureus from Pakistan. Trop J Pharm Res. 2012;11(6):925-31.

34. Maiyo Z, Ngure R, Matasyoh J, Chepkorir R. "Phytochemical constituents and antimicrobial activity of leaf extracts of three Amaranthus plant species. Afr J Biotechnol. 2010;9(21):3178-82.
35. Khan SU, Khan AU, Shah AU, Shah SM, Hussain S, Ayaz M, Ayaz S Heavy metals content, phytochemical composition, antimicrobial and insecticidal evaluation of Elaeagnus angustifolia. Toxicol Ind Health. 2016;32(1):154-61.

36. Zul K, Midrarullah, Sajjad A, Farhat U, Abdul S, Muhammad A, Anwar Z, Imran M. Ex-vivo antibacterial, phytotoxic and cytotoxic, potential in the crude natural phytoconstituents of Rumex hastatus d. Don. Pak J Bot. 2015;47(SI):293-9.

37. Ullah I, Subhan F, Ayaz M, Shah R, Ali G, Haq IU, Ullah S. Anti-emetic mechanisms of zingiber officinale against cisplatin induced emesis in the pigeon; behavioral and neurochemical correlates. BMC Complement Altern Med. 2015;15(1):34.

38. Zeb A, Sadiq A, Ullah F, Ahmad S, Ayaz M. Investigations of anticholinesterase and antioxidant potentials of methanolic extract, subsequent fractions, crude saponins and flavonoids isolated from Isodon rugosus. Biol Res. 2014;47:76.

39. Kamal Z, Ullah F, Ayaz M, Sadiq A, Ahmad S, Zeb A, Hussain A, Imran M. Anticholinesterse and antioxidant investigations of crude extracts, subsequent fractions, saponins and flavonoids of atriplex laciniata L.: potential effectiveness in Alzheimer's and other neurological disorders. Biol Res. 2015;48:1-11.

40. Kumar S, Koh J, Kim H, Gupta M, Dutta P. A new chitosan-thymine conjugate: Synthesis, characterization and biological activity. Int J Biol Macromol. 2012;50(3):493-502

41. Hughes PG, Verge JP. Oxazole and imidazole derivatives as antifungal agents. In: Google Patents. 1975.

42. Zentz F, Le Guillou R, Labia R, Sirot D, Linard B, Valla A. Syntheses, in vitro antibacterial and cytotoxic activities of a series of 3-substituted succinimides. Farmaco. 2004;59(11):879-86.

43. Kumar V, Ahamad T, Nishat N. Some O, O', O ", O"'-di/tetra aryldithioimidophonate transition metal complexes derived from catechol and bisphenol-A as antibacterial and antifungal agents. Eur J Med Chem. 2009;44(2):785-93.

44. Yang D, Michel L, Chaumont J-P, Millet-Clerc J. Use of caryophyllene oxide as an antifungal agent in an in vitro experimental model of onychomycosis. Mycopathol. 2000;148(2):79-82.

45. Kim Y, Park S, Lee E, Cerbo R, Lee S, Ryu C, Kim G, Kim J, Ha Y. Antibacterial compounds from rose Bengal-sensitized photooxidation of $\beta$-caryophyllene. J Food Sci. 2008;73(7):C540-5.

46. Cueva C, Moreno-Arribas MV, Martín-Álvarez PJ, Bills G, Vicente MF, Basilio A Rivas $C L$, Requena T, Rodríguez JM, Bartolomé B. Antimicrobial activity of phenolic acids against commensal, probiotic and pathogenic bacteria. Res Microbiol. 2010;161(5):372-82.

47. Togashi N, Inoue $Y$, Hamashima H, Takano A. Effects of two terpene alcohols on the antibacterial activity and the mode of action of farnesol against Staphylococcus aureus. Molecules. 2008;13(12):3069-76.

48. Saidi S, Luitaud C, Rouabhia M. In vitro synergistic effect of farnesol and human gingival cells against Candida albicans. Yeast. 2006;23(9):673-87.

49. Narasimhan B, Mourya V, Dhake A. Design, synthesis, antibacterial, and QSAR studies of myristic acid derivatives. Bioorg Med Chem Lett. 2006;16(11):3023-9.

50. Parang K, Knaus EE, Wiebe LI, Sardari S, Daneshtalab M, Csizmadia F. Synthesis and antifungal activities of myristic acid analogs. Arch Pharm. 1996:329(11):475-82.

51. Agoramoorthy G, Chandrasekaran M, Venkatesalu V, Hsu M. Antibacterial and antifungal activities of fatty acid methyl esters of the blind-your-eye mangrove from India. Braz J Microb. 2007;38(4):739-42.

52. Maliszewska J, Tęgowska E. Capsaicin as an organophosphate synergist against Colorado potato beetle (Leptinotarsa decemlineata Say). J Plant Prot Res. 2012;52(1):28-34.

53. Arnason J, Philogene B, Morand P. Insecticides of plants origin, vol. 387. Washington: Amer Chemical Soc Symp Series; 1989.

54. Isman M. Plant essential oils for pest diseases management. Crop Prot. 2000; 19:603-8.

55. Francis G, Kerem Z, Makkar H, Becker K. The biological action of saponins in animal systems: a review. J Nutr. 2002;88:587-605.

56. Matthews GC. Insecticide application in stores. In: Matthews GA, Hislop EC, editors. Application technology for crop protection. Wallingford: International; 1993. p. 305-15.

57. Riebeiro B, Guedes R, Oliveira E, Santos J. Insecticide resistance and synergism in Brasilian populations of Sitophilus zeamais (Coleoptera: Curculionidae). J Stored Prod Res. 2003;39:21-31. 
58. Tang $X$, Chen S, Wang L. Isolation and Insecticidal activity of farnesol from Stellera chamaejasme. Asian J Chem. 2011;23(3):1233

59. Wang Y, Wang H, Shen Z, Zhao L, Clarke S, Sun J, Du Y, Shi G. Methyl palmitate, an acaricidal compound occurring in green walnut husks. J Econ Entomol. 2009;102(1):196-202.

60. Siegler E, Popenoe C. The fatty acids as contact insecticides. J Econ Entomol. 1925;18(2):292-9.

Submit your next manuscript to BioMed Central and we will help you at every step:

- We accept pre-submission inquiries

- Our selector tool helps you to find the most relevant journal

- We provide round the clock customer support

- Convenient online submission

- Thorough peer review

- Inclusion in PubMed and all major indexing services

- Maximum visibility for your research

Submit your manuscript at www.biomedcentral.com/submit
Biomed Central 